\title{
A blue-whiting protein hydrolysate induces aminoacidemia in older adults and skeletal muscle anabolism in vitro
}

\author{
M.J. Lees ${ }^{1}$, D. Nolan ${ }^{2}$, B. Egan ${ }^{2}$ and B.P. Carson ${ }^{1,3}$ \\ ${ }^{1}$ Department of Physical Education and Sport Sciences, Faculty of Education and Health Sciences, University of \\ Limerick, Limerick, Ireland, \\ ${ }^{2}$ School of Health and Human Performance, Dublin City University, Dublin, Ireland and \\ ${ }^{3}$ Health Research Institute, University of Limerick, Limerick, Ireland
}

Fish protein represents one of the most widely consumed dietary protein sources by humans (Nations et al, 2012). The processing of material from the fishing industry generates substantial unexploited waste products, many of which possess high biological value. These waste products offer potential as high-quality sources of dietary protein whilst enhancing economic and environmental sustainability. Protein hydrolysates, such as fish protein hydrolysates (FPH), containing predominantly di- and tripeptides, are more readily absorbed than free amino acids and intact protein (He et al., 2013). Furthermore, in animal models FPH have been shown to possess pluripotent beneficial properties for cardiovascular, neurological, intestinal, renal, and immune health (Lees and Carson, 2020). Ageing is associated with the loss of skeletal muscle mass and function (Mitchell et al., 2013). Thus, there appears to be a potential application for FPH in older persons as a high-quality protein source that may also confer additional health benefits. However, there remains a lack of research on the impact of FPH on health outcomes in humans, the mechanisms through which they may act and a comparison with other industry benchmark protein sources.

This study investigated the impact of a blue whiting-derived protein hydrolysate (BWPH) on aminoacidaemia in vivo and skeletal muscle anabolism in vitro compared with whey protein isolate (WPI) and an isonitrogenous non-essential amino acid (NEAA) control $(0.33 \mathrm{~g} \cdot \mathrm{kg}-1 \cdot$ body mass- 1$)$ in an ex vivo, in vitro experimental design. Following ethical approval and obtaining informed consent, human serum was obtained from seven healthy older adults (two males, five females; age: $72 \pm 5$ years, body mass index: $24.9 \pm 1.6 \mathrm{~kg} \cdot \mathrm{m} 2)$ in three separate trials in a randomised, counterbalanced, double-blind design. C2C12 myotubes were treated with ex vivo human serum-conditioned media $(20 \%)$ for $4 \mathrm{~h}$. Anabolic signalling (phosphorylation of mTOR, p70S6K, and 4E-BP1) and puromycin incorporation were determined by immunoblotting. One and two-way repeated measures analysis of variance (ANOVA) were used where appropriate and Holm-Bonferroni adjustment for post-hoc multiple comparisons.

BWPH and WPI both induced postprandial essential aminoacidaemia in older adults above the NEAA control. Increases in leucine and essential amino acids were more pronounced following WPI ingestion compared to BWPH. Muscle protein synthesis as measured by puromycin incorporation was increased in response to both $\mathrm{BWPH}(\mathrm{P}=0.030)$ and WPI $(\mathrm{P}=0.028)$ compared to NEAA. Myotube growth was greater in BWPH and WPI compared with NEAA (both $\mathrm{P}=0.05$ ) but was similar between test conditions $(\mathrm{P}=0.853)$.

These findings demonstrate the anabolic potential of BWPH in vivo and ex vivo, thus providing justification for larger studies in older adults using gold-standard measures of acute and chronic MPS in vivo.

\section{References}

1. Nations F \& A.O. of the U. (2013) Choice Res 50, 50-5350.

2. He S, Franco C \& Zhang W (2013) Food Res Int 50, 289-297.

3. Lees MJ \& Carson BP (2020) Nutrients 13;12(8), 2434.

4. Mitchell WK, Williams J, Atherton P, et al. (2012) Front Physiol. 